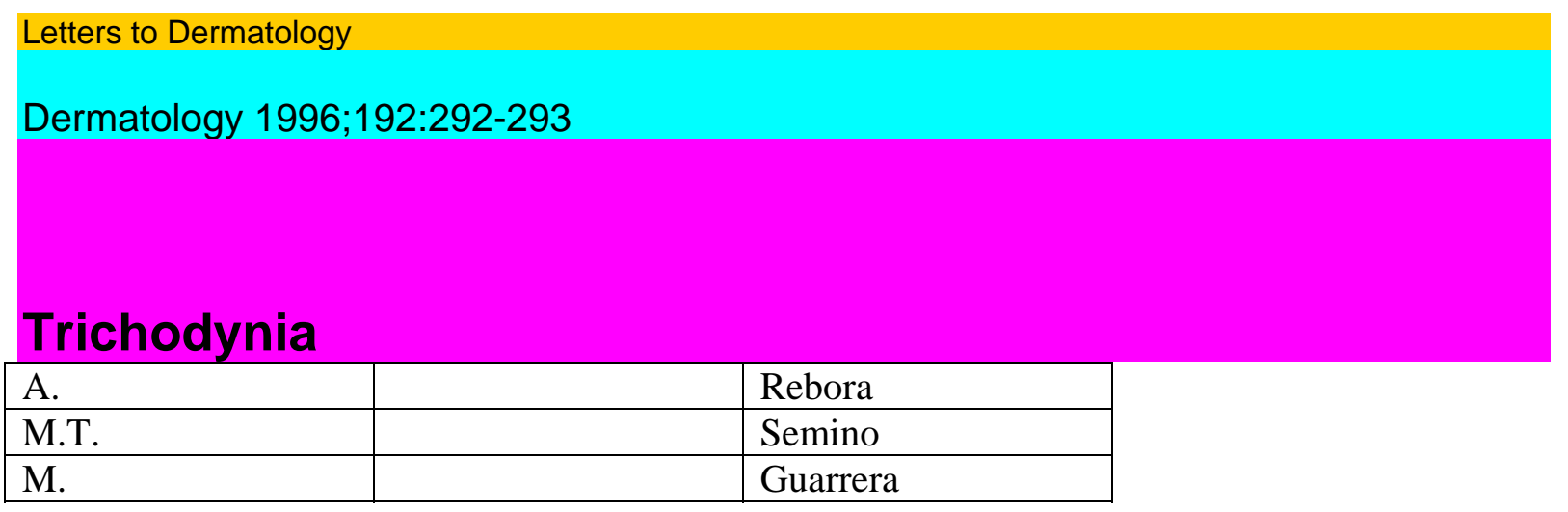

Department of Dermatology, University of Genoa, Italy

\title{
Key Words
}

Baldness

Hair loss

Trichodynia

Dr. Alfredo Rebora, Department of Dermatology, University of Genoa, Viale Benedeno XV, I-16132 Genova (Italy)

No symptoms have ever been reported to accompany the development of baldness. There are patients, however, who, while complaining of hair loss, claim that their hair has become painful. We describe such discomfort and we propose the term trichodynia for it. (The term trichodynia was coined by C. Del Forno, MD, in April 1995.) Two hundred and twenty-two consecutive women seeking advice for hair loss were examined. In particular, they were asked about any discomfort, pain or paresthesia in their scalp or hair. Pruritus was not considered. Hair loss was evaluated by the pull test, daily count and wash test. In brief, 5 days after shampooing, hairs were 'combed' by fingers and the number of hairs remaining in between them was registered. Hairs found on the pillow, comb and shoulders during the day were also counted (daily count). Five days after shampooing, hair was shampooed in a sink whose hole had been covered by a gauze and the number of hairs remaining in the gauze was recorded. A trichogram was also made according to the well-known procedure.

Seventy-six women (34.2\%) complained of trichodynia. Most commonly the symptom was described 'as if the hairs were bent in the wrong way', more rarely as 'stitches'. There was no difference of age between patients with trichodynia and those without (37.3 and 37.7 years, respectively). No relationship was found between the onset of trichodynia and onset of hair loss. In addition, patients with trichodynia did not show a severer hair loss than those without. In particular, with the wash test, patients with trichodynia averaged 207 hairs (median 150) versus 173 hairs (median 120) counted by those without ( $\mathrm{t}=1.345 ; \mathrm{p}>0.05$ ) and $68 \%$ of patients with trichodynia counted 100 or more hairs versus $57 \%$ of those without (Fisher's test $p=0.007$ ). Also, the daily count, the pull test and the percentage of telogen hairs showed no difference. Lastly, there was no relationship with the severity of hair thinning, $17 \%$ of patients with trychodinia having Ludwig alopecia grades I-III versus $21 \%$ of patients without. In women complaining of hair loss, it is always difficult to discriminate between reality and delusion. In doubtful cases, most dermatologists tend to favour the second choice. We decided to give credit to the claim and took to the idea that hair loss is painful in about one third of patients.

292 Dermatology 1996;192:292-293

Letters to Dermatology 
In the absence of any relationship with other parameters of hair loss and any histopathological data, however, it is difficult to understand the reasons of such a symptom. Inflammation may be suspected, as in androgenetic alopecia a perifollicular lymphocytic infiltrate has been described and credited of pathogenetic importance [1].

Reference 1 Jaworsky C, Kligman AM, Murphy GF: Characterization of inflammatory infiltrate in male pattern alopecia: Implications for pathogenesis. Br J Der-matol 1992;127:239-246.

Dermatology 1996:192:293-294

Familial Background in Aberrant Mammary Tissue Is a 'Protective' Factor against the Development of Nephrourinary Anomalies

CE. Urbani”, R. Betti”

“Service of Dermatology, San Raffaele 'Resnati' Hospital, and bIVth Dermatologic Clinic, San Paolo Hospital, Milan, Italy

Key Words

Aberrant mammary tissue $\cdot$ Nephrourinary malformation •

Polythelia

In our previous survey, we discussed the clinicoepidemiological features regarding familial aberrant mammary tissue (AMT) by investigating 18 pedigrees out of a population of 156 adult Caucasian subjects [1], Among the main questions concerning familial AMT we also briefly debated the problem about its association with kidney and urinary tract malformations (KUTM). Then, we concluded that paper with the following words: 'However, in opposition to the sporadic forms, familial AMT, in our experience, seems to be unrelated to an increasing frequency of this association' [1]. During the past 15 months we have observed and extensively studied 10 further pedigrees - recorded, as previously specified [ $1 \mathrm{~J}$, by using a questionnaire and, when available, by direct examination of family members (doubtful cases were not included) - in order to further investigate the association between familial AMT and KUTM. The instrumental investigations (complete abdominal and renal ultrasound) and the laboratory tests (blood examinations, urinalysis and cultures) invariably excluded the evidence of clinically overt or occult renal anomalies in all this second series of 10 patients. Therefore, in 28 cases of familial AMT that we have examined on the whole, no KUTM has been demonstrated.

Our results agree with those of Bortz et al. [ 2 ] who did not disclose any association between familial polythelia and renal anomalies by investigating, however, only one family of 4 (parents and 2 sons) affected with a left-sided supernumerary nipple. Conversely, in another report by Goedert et al. [3], 2 patients of 6 showed an overlap between familial polythelia, kidney malformations and renal adeno-carcinoma. Similarly, Toumbis-Ioannou and Cohen [41 described 3 siblings ( 2 sisters and 1 brother) affected with left-sided polythelia and polymastia; the younger of them had also a concomitant ectopic

right kidney [4]. To the best of our knowledge, in the literature there are no other studies dealing with this specific issue.

The lack of evidence of KUTM we have also observed in this second series of familial AMT could reflect the presence of some genetic factors involved in the pathogenetic mechanisms of the mammore-nal association. The male-to-male pattern of inheritance recorded in about $75 \%$ of them $[1,5]$ could support the existence of a peculiar mode of inheritance within familial AMT. According to the embryo developmental field defect concept [6], the mammorenal association reflects, in fact, a common disturbance of the fetus in the so-called supernumerary nipple/renal field [7]. We may hypothesize that such genetic transmission could influence this association 
and, furthermore, prevent those 'noxae' from acting during embryogenesis inside the polytopic fields involved and, finally, from causing concomitant nephrourinary abnormalities.

On the other hand, it has been well documented that the association between AMT and KUTM is greatly influenced by racial [8], and ethnic and geographic differences [9]. Rahbar [10] and Robertson et al. [8] have recorded no renal anomalies in black neonates and infants with supernumerary nipples, Hungarian authors $[11,12]$ found a very high prevalence of KUTM in their pediatric series, while different conclusions are reported from Israel where various ethnic origins are present $[9,13]$.

By evaluating our results, the presence of a familial background in the subjects affected with AMT may be considered a further intrinsic protective factor against the development of congenital and hereditary nephrourinary defects.

These data are conflicting if compared with those concerning the sporadic forms of AMT that are characterized, on the contrary, by a significant association with KUTM [14] (presently: 16/167, $9.58 \%)$.

Therefore, the detection of a patient presenting with AMT should always alert the physician for a careful approach - including also a detailed family history - and prompt complete abdominal ultrasound examination to exclude early the occurrence of KUTM.

References

Urbani CE, Betti R: Familial aberrant mammary tissue: A clinicoepidemiological survey of 18 cases. Dermatology 1995;190:207-209.

Bortz J, Parker S, Ray TL: Lack of associated renal anomalies in familial polythelia (letter). Am J Dis Child 1989;43:883.

Goedert JJ, McKeen EA, Fraumeni JF Jr: Polymastia and renal adenocar-cinoma. Ann Intern Med 1981;95:182-184.

Toumbis-Ioannou E, Cohen PR: Familial polythelia. J Am Acad Dermatol 1994;30:667-668. Urbani CE. Betti R: The significance of familial polythelia (letter). J Am Acad Dermatol 1995;32:687-688.

Opitz JM: The developmental field concept in clinical genetics. J Pediatr 1982;101:805-809. Hersh JH, Bloom AS, Cromer O. Harrison HL, Weisskopf B: Does a supernumerary nipple/renal field defect exist? Am J Dis Child 1987; 141: 989-991.

Robertson A, Sale P, Sathyanarayan: Lack of association of supernumerary nipples with renal anomalies in black neonates. J Pediatr 1986; 109: 502-503.

Varsano IB, Jaber L, Garty BZ, Mukamel MM. Grunebaum M: Urinary tract abnormalities in children with supernumerary nipples. Pediatrics 1984;73:103-105.

Rahbar F: Clinical significance of supernumerary nipples in black neonates. Clin Pediatr 1982;21:46-47.

Méhes K: Association of supernumerary nipples with other anomalies. J Pediatr 1979;95:274275.

Meggyessy V, Méhes K: Association of supernumerary nipples with renal anomalies. J Pediatr 1987;Ill:412-413.

Letters to Dermatology

Dermatology 1996:192:292-295 293 